\title{
EDITORIAL
}

\section{Special issue: Health promoting activities of phytochemicals}

\author{
Anat Elmann \\ Department of Food Science, Volcani Center, Agricultural Research Organization, Rishon Lezion, 7505101, Israel
}

Traditionally, horticultural research has concentrated on plant biochemistry and physiology, agricultural aspects, crop resistance to diseases, and so on. However, all plants contain many and various phytochemicals, with diverse biological activities. Throughout most of human history, before the development of the conventional pharmaceutical industry, herbal extracts containing these bioactive molecules were used to treat various diseases and symptoms. Herbal substances have also inspired the development of conventional medicines, with some of these medicines requiring a doctor's prescription. The process of discovering plant substances with medical activities is long and multistaged, and is based on both ethnobotanical knowledge and experimental evidence. Due to the development of new areas of science, scientists have gained better understanding regarding the potential of plants beyond these classical research areas. This is why horticultural research expanded to new areas that bridge between human health and horticulture, the horticulture-health interphase.

\section{Research papers}

The articles in this special issue include twelve research papers along with five review articles. The research papers in this issue discuss newly discovered biological activities of phytochemicals and plant extracts that can be potentially developed as treatments for various disorders, and the underlying mechanisms by which they affect biological systems.

\section{COVID-19}

The COVID-19 pandemic which is the current global health challenge is also represented in this issue by an in-silico study (Sharma et al.) and by a review article (Singh et al.) summarizing the possible role of traditional medical systems to manage COVID-19.

\section{Cancer}

A research article, by Kirbag et al., demonstrates the effects of different plants and their bioactive compounds on cancer cell lines. A review article by Azhagumeena et al. describes the cancer therapeutic potential of Piper betle, Syzygium aromaticum and their bioactive component eugenol.

\section{Skin disorders}

Three articles, by Zhou et al., Wang et al., and Telerman et al., describe the effects of plant extracts and purified phytochemicals (isoliquiritigenin, luteolin and achillolide), in vivo and in cellular systems, on skin disorders such as atopic dermatitis and ischemia-reperfusion injury.

\section{Allergic rhinitis}

Wang et al. demonstrate the protective effect of ellipticine in a murine model for allergic rhinitis. 


\section{Oxidative stress and inflammation}

Four articles, by Feng et al., Li et al., Zhou et al. and Telerman et al., highlight the ability of phytochemicals to reduce oxidative stress and inflammation, two major processes which are involved in most pathological conditions. Feng et al. present the protective effect of the phytochemical Boldine against neuroinflammation in spinal cord injury in rats, Wang et al. show the effect of ellipticine in a murine model for allergic rhinitis, Telerman et al. demonstrate the effect of achillolide $A$ in atopic dermatitis, and Li et al. present the inhibitory effect of Xuebijing in human synovial cells. Two more articles discuss the in vitro antioxidant activities of some Indigofera species (by Kolar et al.) and of Thai formulated polyherbal tea (Adekoya et al.).

\section{Signal transduction}

Two research articles demonstrate the effect of an intravenous preparation made from five traditional Chinese herbal medicines in human synovial cells ( $\mathrm{Li}$ et al.), and a purified phytochemical (ellipticine) in an ovalbumin (OVA)-induced murine model of allergic rhinitis (Wang et al.) on signaling pathways and on the transcription factor, NFkB.

\section{Chemical analyses}

It is highly important to chemically identify and quantify the compounds present in bioactive plant extracts.
Two articles, by Ananth et al. and by Sharma et al., analyze the chemical composition of the volatiles and phenolic compounds in Cassia auriculata and Clinopodium serpyllifolium.

\section{Review articles}

The five review articles in this issue discuss the ethnobotany and the usage of five plants in traditional and modern medicine, and describe the phytochemicals responsible for the bioactivities of cannabis (Ofir), jojoba (Tietel et al.), Barberry (Sun et al.), Piper betle and Syzygium aromaticum (Azhagumeena et al.). Another review article (Singh et al.) summarizes the available scientific data supporting the use of traditional medicine for prevention and treatment of COVID-19.

\section{Dedication}

I would like to dedicate this special issue to two research pioneers in the field of medicinal plants in Israel. Prof. Zohara Yaniv-Bachrach (from the Volcani Institute) and Dr. Mina Paran, who were engaged in the ethnobotany, chemistry and bioactivities of medicinal plants. Each of them were teaching students and professionals, writing and researching medicinal herbs in Israel. In 2003, Prof Zohara Yaniv-Bachrach and Dr. Mina Paran were among the founders of the Israeli Association of Medicinal Plants. 